\title{
Set-up errors due to endorectal balloon positioning in intensity modulated radiation therapy for prostate cancer
}

\author{
Chun-Wei Wang ${ }^{\mathrm{a}, \mathrm{b}, \mathrm{d}}$, Fok-Ching Chong ${ }^{\mathrm{a}}$, Ming-Kuen Lai ${ }^{\mathrm{b}, \mathrm{c}, \mathrm{d}}$, Yeong-Shiau Pu \\ Jian-Kuen Wu ${ }^{\mathrm{b}}$, Jason Chia-Hsien Cheng ${ }^{\mathrm{b}, \mathrm{d}, \mathrm{e}, *}$ \\ anstitute of Electrical Engineering, National Taiwan University, ${ }^{\mathrm{b}}$ Department of Oncology, and ${ }^{\mathrm{C} D e p a r t m e n t}$ of Urology, National \\ Taiwan University Hospital, Taipei, Taiwan, ${ }^{\mathrm{d}}$ Cancer Research Center, and ${ }^{\mathrm{e}}$ Graduate Institute of Clinical Medicine, National \\ Taiwan University College of Medicine, Taipei, Taiwan
}

\begin{abstract}
Purpose: To investigate the set-up errors and deformation associated with daily placement of endorectal balloons in prostate radiotherapy.

Materials and methods: Endorectal balloons were placed daily in 20 prostate cancer patients undergoing radiotherapy. Electronic portal images (EPIs) were collected weekly from anterior-posterior (AP) and lateral views. The EPIs were compared with digitally reconstructed radiographs from computed tomography scans obtained during pretreatment period to estimate displacements. The interfraction deformation of balloon was estimated with variations in diameter in three orthogonal directions throughout the treatment course.

Results: A total of $154 \mathrm{EPIs}$ were evaluated. The mean displacements of balloon relative to bony landmark were $1.8 \mathrm{~mm}$ in superior-inferior (SI), $1.3 \mathrm{~mm}$ in AP, and $0.1 \mathrm{~mm}$ in left-right (LR) directions. The systematic errors in SI, AP, and LR directions were $3.3 \mathrm{~mm}, 4.9 \mathrm{~mm}$, and $4.0 \mathrm{~mm}$, respectively. The random (interfraction) displacements, relative to either bony landmarks or treatment isocenter, were larger in SI direction $(4.5 \mathrm{~mm}$ and $4.5 \mathrm{~mm})$, than in AP $(3.9 \mathrm{~mm}$ and $4.4 \mathrm{~mm})$ and LR directions $(3.0 \mathrm{~mm}$ and $3.0 \mathrm{~mm})$. The random errors of treatment isocenter to bony landmark were $2.3 \mathrm{~mm}, 3.2 \mathrm{~mm}$, and $2.6 \mathrm{~mm}$ in SI, AP, and LR directions, respectively. Over the treatment course, balloon deformations of $2.8 \mathrm{~mm}, 2.5 \mathrm{~mm}$, and $2.6 \mathrm{~mm}$ occurred in SI, AP, and LR directions, respectively. The coefficient of variance of deformation was $7.9 \%, 4.9 \%$, and $4.9 \%$ in these directions.
\end{abstract}

Conclusions: Larger interfractional displacement and the most prominent interfractional deformation of endorectal balloon were both in SI direction.

๑) 2007 Elsevier Ireland Ltd. All rights reserved. Radiotherapy and Oncology 84 (2007) 177-184.

Keywords: Prostate cancer; Intensity modulated radiation therapy; Endorectal balloon; Setup error; Deformation

The role of definitive radiotherapy for localized prostate cancer is well established. Many studies of radiation dose escalation for better local control of prostate cancer have been published in recent decades [5,10,22,23]. The results from retrospective analysis or prospective randomized trials all showed that dose escalation improved biochemical control in patients with localized prostate cancer. Notably, in a phase III randomized trial, dose escalation was associated with higher incidence of late rectal toxicity [10]. At 6 years, grade 2 or higher rectal toxicity rates were $26 \%$ in the doseescalation group ( $78 \mathrm{~Gy}$ ) compared with $12 \%$ in the conventional-dose group (70 Gy).

Several methods to reduce late rectal toxicity have been investigated in prostate radiotherapy. The intensity modulated radiation therapy (IMRT) technique significantly reduced the incidence of late grade 2 rectal toxicity compared with three-dimensional conformal radiotherapy [22]. Another strategy is to reduce the rectal volume within the high-dose region, given that incidence of rectal complications correlates with radiation dose and irradiated volume of tissue [12,13]. Endorectal balloons, designed for the purpose of rectal sparing, have been used in prostate radiotherapy at some institutes. They significantly reduce the rectal volume receiving high-dose prostate irradiation [9]. Besides, prostate immobilization is improved because the endorectal balloon pushes the prostate toward the pubic symphysis. Other studies have demonstrated that endorectal balloons reduce both the prostate movement and variation in rectum filling during treatment $[3,21]$.

However, another investigation found endorectal balloons had the opposite effect on prostate immobilization [20] and failed to reduce interfraction variation. This 
contradictory finding might be explained by the presence of stool or gas in the rectum adjacent to balloon. Extrinsic factors (such as stool, gas, and tension in the pelvic cavity) would cause inconsistent balloon positioning and deformation of the balloon. Moreover, positioning error associated with daily balloon placement may be another issue of concern. To date, the consistency of endorectal balloon placement has seldom been addressed. We conducted this study to investigate the set-up error due to endorectal balloon positioning, and compare systematic (present during all treatment fractions) and random (interfraction) variation. We also evaluated the deformation of the endorectal balloon during daily placement throughout the course of intensity modulated radiation therapy (IMRT).

\section{Materials and methods}

\section{Treatment and patient setup}

Twenty patients with prostate cancer undergoing IMRT at National Taiwan University Hospital were enrolled in this study. Patient characteristics are shown in Table 1. All patients were treated with 10-MV photon beam from a Siemens Primus Linear Accelerator. Five gantry angles with a total of $35-50$ segments were used for the IMRT. The margins for planning target volume (PTV) were $0.6 \mathrm{~cm}$ in posterior (toward rectum) direction, and $1.0 \mathrm{~cm}$ in the other directions. A total dose of $78 \mathrm{~Gy}$ in 39 daily fractions, five fractions per week, was given to the PTV. The $100 \%$ prescription isodose curve encompassed al least $95 \%$ of PTV in all cases. Less than $5 \%$ of PTV received more than $110 \%$ of

\begin{tabular}{|c|c|c|}
\hline Characteristics & Number & $\%$ \\
\hline \multicolumn{3}{|l|}{ Age at diagnosis } \\
\hline Mean & 73.8 & \\
\hline$<75$ & 9 & 45 \\
\hline$\geqq 75$ & 11 & 55 \\
\hline \multicolumn{3}{|l|}{ T stage } \\
\hline $\mathrm{T} 1$ & 3 & 15 \\
\hline $\mathrm{T} 2$ & 5 & 25 \\
\hline T3 & 11 & 55 \\
\hline T4 & 1 & 5 \\
\hline \multicolumn{3}{|l|}{$\mathrm{N}$ stage } \\
\hline NO & 20 & 100 \\
\hline N1 & 0 & 0 \\
\hline \multicolumn{3}{|l|}{ Pre-treatment PSA $(\mathrm{ng} / \mathrm{ml})$} \\
\hline$<10$ & 7 & 35 \\
\hline $10-20$ & 7 & 35 \\
\hline$>20$ & 6 & 30 \\
\hline \multicolumn{3}{|l|}{ Gleason score } \\
\hline$<7$ & 6 & 30 \\
\hline$=7$ & 7 & 35 \\
\hline$>7$ & 7 & 35 \\
\hline \multicolumn{3}{|l|}{ Treatment target } \\
\hline Prostate alone & 6 & 30 \\
\hline Prostate + bilateral seminal vesicles & 14 & 70 \\
\hline
\end{tabular}

prescription dose. The constraints for bladder and rectum were less than $25 \%$ and $17 \%$ volume receiving doses of more than $65 \mathrm{~Gy}$, respectively. The isodose curves of one representative patient's plan in transverse and sagittal images are shown in Fig. 1. During the simulation and whole course of IMRT, the patients were immobilized in the prone position with a vacuum bag. Our in-house protocol did not include the insertion of gold markers into the prostate.

\section{Placement of endorectal balloon}

Daily placement of the endorectal balloon was performed under uniform guidelines for prostate immobilization and rectal sparing. One endorectal balloon was used per patient throughout the entire treatment course with the use of a disposable condom in each fraction. The deflated endorectal balloon was inserted into the rectum, inflated with $60 \mathrm{ml}$ of air [9], and gently pulled toward the anal sphincter. In simulation, a marking sign was made on the endorectal balloon shaft at the level of anal verge. To ensure the reproducible positioning of endorectal balloon, the placement of balloon needs the correction of fitting the marking sign position at anal verge in daily procedure. The marking sign on the balloon shaft was made to ensure the relatively constant position of the balloon in daily placement, but not for the correction of treatment position. In daily pre-treatment set-up, positioning was based on the skin marker of the patient. All patients tolerated the procedure well throughout the treatment course.

\section{Image collection}

Electronic portal images (EPIs) were collected weekly from anterior-posterior (AP) and lateral views for each patient. The EPIs were compared with digitally reconstructed radiographs (DRR) of computed tomography scans from pretreatment simulations to evaluate the set-up errors in distance between the endorectal balloon, bony landmarks, and treatment isocenter. The reference point of the endorectal balloon was the center of the air sac in the catheter before inflation. In the lateral view, the reference points of bony landmarks were the apex of the pubic symphysis and the anterior sacral edge at the level of the tip of the femoral head. In the AP view, the reference point was the midpoint between the tips of the bilateral femoral heads. The set-up errors in endorectal balloon positioning were measured from the balloon center to the bony landmarks and treatment isocenter. Displacement of the treatment isocenter relative to the bony landmarks was also measured to evaluate the interfraction positioning variation during the course of IMRT. All measurements were collected in three orthogonal directions [superior-inferior (SI), anterior-posterior (AP), and left-right (LR)] separately. To evaluate the deformation of the endorectal balloon in daily placement, the diameters of the endorectal balloon in the $\mathrm{SI}$, $\mathrm{AP}$, and LR directions were measured from the EPIs for each patient.

\section{Data analysis}

The systematic and random errors in the displacement of the endorectal balloon relative to the bony landmarks and 

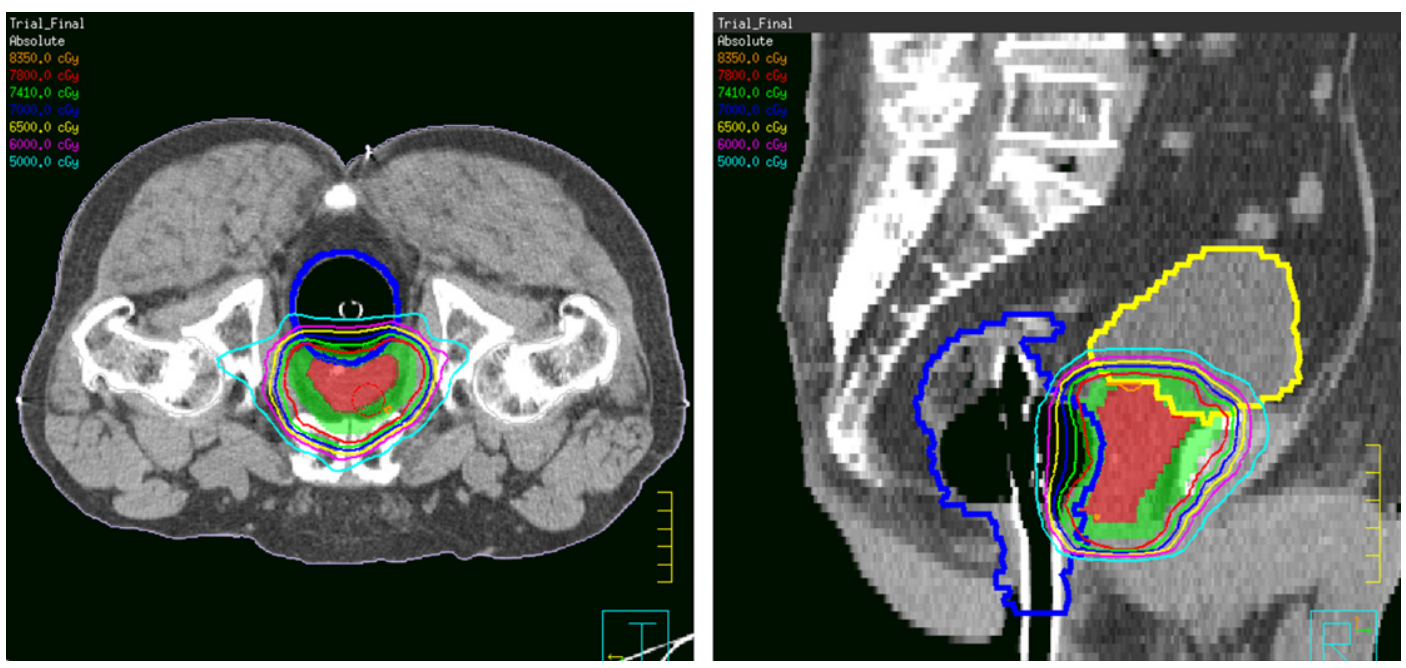

Fig. 1. The isodose curves of one representative patient's plan in transverse and sagittal images.

treatment isocenter were estimated. The systematic error was defined as the standard deviation (SD) of the average displacement for each patient in the whole group, representing the variation in the pre-existing displacement between the simulation and daily treatment set-up $[14,16]$. The random error was defined as the root mean square of SD of displacement for each patient in the whole group, indicating the random fraction-to-fraction (interfraction) variations of balloon placement. The deformation of the endorectal balloon was assessed by calculating the SD and coefficient of variance of diameter change in three orthogonal directions. Linear regression analysis was done for the correlation between the deformation and displacement of the endorectal balloon in three directions. $R^{2}$ represented the correlation coefficient. All tests were two-tailed and $p$ value of less than 0.05 was considered significant.

\section{Results}

The displacement of the endorectal balloon relative to bony landmarks

The means and SDs of measurements from 154 EPIs were calculated. The center of endorectal balloon was a mean of $13.5 \mathrm{~mm}$ inferior to the apex of pubic symphysis, $44.9 \mathrm{~mm}$ anterior to the sacral point, and $0.5 \mathrm{~mm}$ lateral to the midpoint between the tips of the bilateral femoral heads. One representative case is shown in Fig. 2. For the whole group, the mean displacements were all less than $3 \mathrm{~mm}$ in three orthogonal directions (Table 2). The systematic errors in balloon displacement relative to bony landmarks were $3.3 \mathrm{~mm}$ in the SI direction, $4.9 \mathrm{~mm}$ in the AP direction, and $4.0 \mathrm{~mm}$ in the LR direction. The random error was larger in the SI direction $(4.5 \mathrm{~mm})$ as compared to the AP $(3.9 \mathrm{~mm})$ and LR $(3.0 \mathrm{~mm})$ directions. Random (interfraction) errors
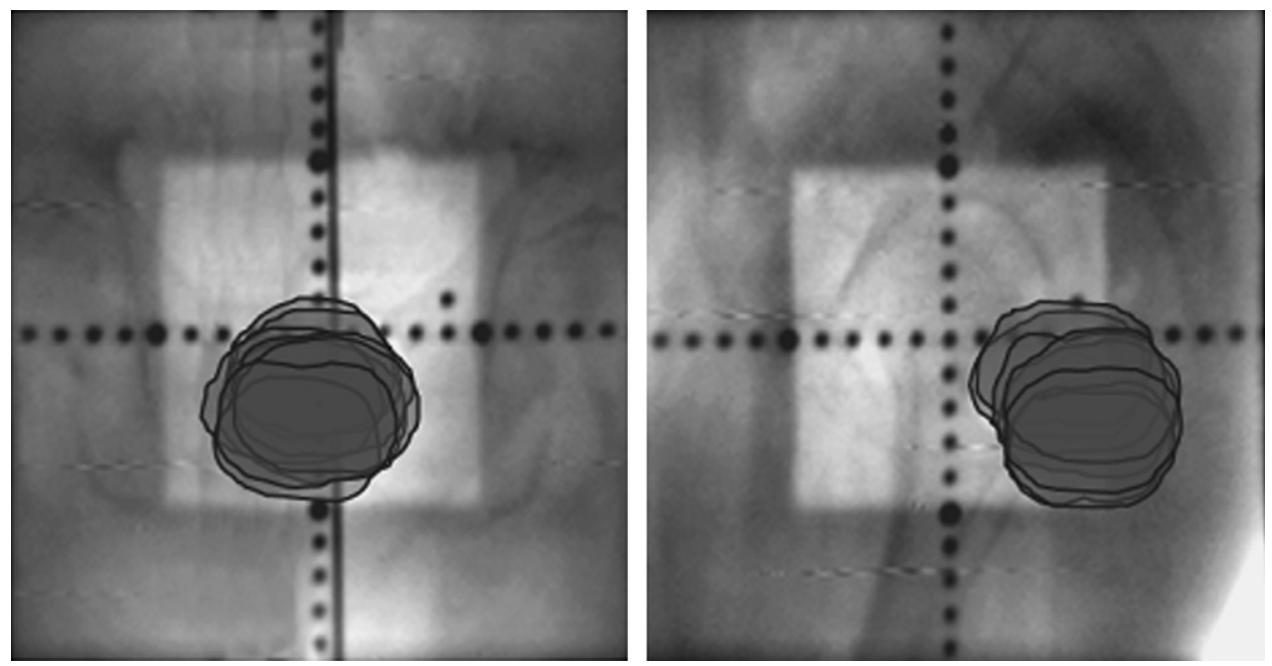

Fig. 2. Changes in position and shape of endorectal balloon on weekly electronic portal images for one representative patient in anteriorposterior (left) and lateral (right) views. 
Table 2

The displacements between balloon center, bony landmarks, and treatment isocenter

\begin{tabular}{lccll}
\hline & $\begin{array}{l}\text { Mean } \\
(\mathrm{mm})\end{array}$ & $\begin{array}{l}\text { Range } \\
(\mathrm{mm})\end{array}$ & $\begin{array}{l}\text { Systematic } \\
\text { error } \\
(1 \mathrm{SD}, \\
\mathrm{mm})\end{array}$ & $\begin{array}{l}\text { Random } \\
\text { error } \\
(1 \mathrm{SD}, \\
\mathrm{mm})\end{array}$ \\
\hline Balloon-Bone & & & & \\
SI & 1.8 & $-4.4 \sim 10.5$ & 3.3 & 4.5 \\
AP & 1.3 & $-11.8 \sim 8.9$ & 4.9 & 3.9 \\
LR & 0.1 & $-4.3 \sim 12.4$ & 4.0 & 3.0 \\
Balloon-Isocenter & & & & \\
SI & 4.4 & $-7.3 \sim 14.3$ & 5.2 & 4.5 \\
AP & 1.1 & $-11.4 \sim 11.0$ & 6.1 & 4.4 \\
LR & 1.5 & $-7.5 \sim 6.1$ & 3.4 & 3.0 \\
Isocenter-Bone & & & & \\
SI & 1.9 & $-7.4 \sim 2.5$ & 2.9 & 2.3 \\
AP & 3.4 & $-9.7 \sim 2.4$ & 3.3 & 3.2 \\
LR & 2.5 & $-1.9 \sim 15.7$ & 4.0 & 2.6 \\
\hline
\end{tabular}

Abbreviations: AP, anterior-posterior; LR, left-right; SI, superior-inferior; SD, standard deviation.

larger than $5 \mathrm{~mm}$ were observed in 7 patients (35\%) for the SI direction, 3 patients (15\%) for the AP direction, and 0 patients for the LR direction. The sequential variation in average displacement of the endorectal balloon in three orthogonal directions for all 20 patients during the IMRT course is shown in Fig. 3a.

\section{The displacement of the endorectal balloon relative to the treatment isocenter}

The balloon center was located a mean of $2.9 \mathrm{~mm}$ inferior to, $30.5 \mathrm{~mm}$ posterior to, and $4.0 \mathrm{~mm}$ lateral to the treatment isocenter. For the whole group, the mean displacement on EPIs relative to DRR from simulation CT was less than $3 \mathrm{~mm}$ in the AP and LR directions, except in the $\mathrm{SI}$ direction. The systematic errors in balloon displacement relative to the treatment isocenter were $5.2 \mathrm{~mm}$ in the $\mathrm{SI}$ direction, $6.1 \mathrm{~mm}$ in the AP direction, and $3.4 \mathrm{~mm}$ in the LR direction (Table 2). Similarly, a larger random (interfraction) error was observed in the SI direction $(4.5 \mathrm{~mm})$ as compared to the AP $(4.4 \mathrm{~mm})$ and LR $(3.0 \mathrm{~mm})$ directions. The random errors larger than $5 \mathrm{~mm}$ were seen in 5 patients (25\%) for the SI direction, 5 patients $(25 \%)$ for the AP direction, and 1 patient (5\%) for the LR direction. The sequential variation in average displacement of the endorectal balloon in three orthogonal directions for the 20 patients during the IMRT course is shown in Fig. 3b.

\section{The interfraction motion of the isocenter relative to bony landmarks}

As listed in Table 2, the average displacement of the treatment isocenter relative to the bony landmarks was less than $3 \mathrm{~mm}$ in the SI and LR directions, but not in the AP direction $(3.4 \mathrm{~mm})$. The systematic and random errors were $2.9 \mathrm{~mm}$ and $2.3 \mathrm{~mm}$ in the SI direction, $3.3 \mathrm{~mm}$ and $3.2 \mathrm{~mm}$ in the AP direction, and $4.0 \mathrm{~mm}$ and $2.6 \mathrm{~mm}$ in the $\mathrm{LR}$ direction, respectively. For the fraction-to-fraction variation, the errors in the AP direction appeared to be larger than
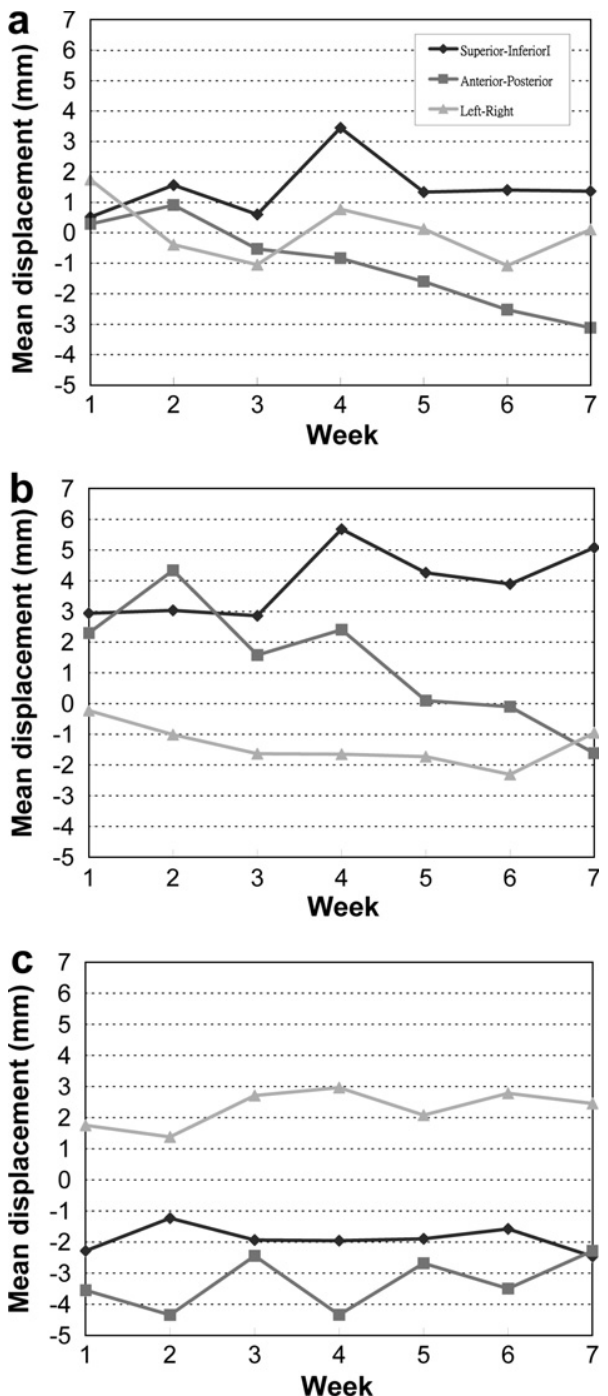

Fig. 3. The sequential displacement between (a) balloon and bony landmarks; (b) balloon and treatment isocenter; (c) treatment isocenter and bony landmarks during the whole course of treatment.

in the other directions. The sequential variation in the average displacement of the isocenter over the treatment period is displayed in Fig. 3c.

\section{The deformation of the endorectal balloon}

The mean diameters of the endorectal balloon were $35.3 \mathrm{~mm}, 51.1 \mathrm{~mm}$, and $53.5 \mathrm{~mm}$ in the SI, AP, and LR directions, respectively. A relatively larger variation in balloon diameter was observed in the SI direction $(2.8 \mathrm{~mm}, 1 \mathrm{SD})$ as compared to that in the AP $(2.5 \mathrm{~mm}, 1 \mathrm{SD})$ and LR (2.6 mm, $1 \mathrm{SD})$ directions (Table 3 ). The coefficient of variance of balloon deformation was $7.9 \%$ in the SI, $4.9 \%$ in the AP, and $4.9 \%$ in the LR directions. Half of our patients had the greatest variation in the SI direction. In linear regression analysis, no significant correlations between deformation and displacement of the endorectal balloon were observed in the SI $\left(R^{2}=0.12, p=0.14\right), \mathrm{AP}\left(R^{2}=0.02\right.$, $p=0.80)$, and LR $\left(R^{2}=0.07, p=0.42\right)$ directions. The devia- 
Table 3

The variation in diameter of endorectal balloon

\begin{tabular}{llll}
\hline & Mean $(\mathrm{mm})$ & SD $(\mathrm{mm})$ & Range $(\mathrm{mm})$ \\
\hline SI & 35.3 & 2.8 & $27.9-42.0$ \\
AP & 51.1 & 2.5 & $41.2-58.5$ \\
LR & 53.5 & 2.6 & $46.6-60.0$ \\
\hline
\end{tabular}

Abbreviations: AP, anterior-posterior; LR, left-right; SI, superior-inferior; SD, standard deviation.

tion in diameter change in the three orthogonal directions for each patient is shown in Table 3. Fig. 4 shows the distribution of balloon center displacements on $154 \mathrm{EPIs}$ relative to the treatment isocenter.

\section{Discussion}

The endorectal balloon has been used for a few years to immobilize the prostate during prostate radiotherapy. Many
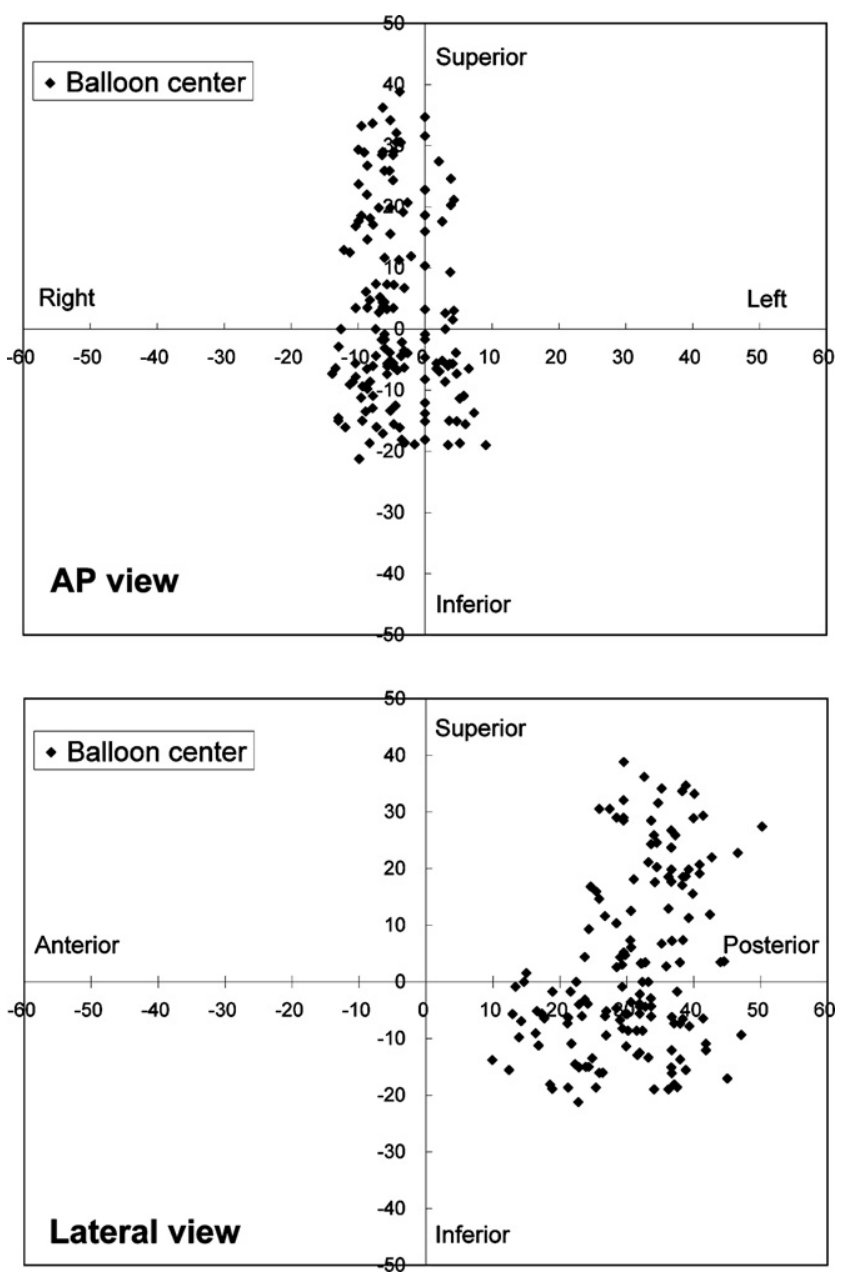

Fig. 4. The distributions of balloon center relative to treatment isocenter on electronic portal images on anterior-posterior (AP) and lateral views. studies have evaluated its effect on prostate immobilization and rectal sparing. However, few studies have evaluated set-up errors associated with endorectal balloon positioning itself. The application of this device relies on daily implementation of a precise set of instructions and on reproducibly inflating the balloon in the rectum with air. Like radiotherapy (which is associated with daily set-up variations), the use of the endorectal balloon is reasonably associated with some set-up errors. We conducted this pilot study to investigate the systematic (pre-existing) deviation and random (interfraction) deviation in endorectal balloon positioning during the treatment course of IMRT for prostate cancer. Besides, we measured the changes in the diameters of endorectal balloons in three orthogonal directions on EPIs, reflecting the variation in the deformation of endorectal balloons between daily placements.

In our study, the random (interfraction) error in endorectal balloon position relative to the bony landmarks was relatively larger in the $\mathrm{SI}$ direction $(4.5 \mathrm{~mm})$, as compared to the AP $(3.9 \mathrm{~mm})$ and LR $(3.0 \mathrm{~mm})$ directions. Similar findings were observed in the set-up error associated with endorectal balloon position in relation to the treatment isocenter. The random errors were $4.5 \mathrm{~mm}, 4.4 \mathrm{~mm}$, and $3.0 \mathrm{~mm}$ in the $\mathrm{SI}, \mathrm{AP}$, and LR directions, respectively. Variation in the daily manual placement of endorectal balloons might contribute to this finding. Although instructions for placing the balloon are specific, the depth of catheter insertion into the rectum might be unavoidably different for each placement. These differences result in the higher variation in the SI direction.

The variation in endorectal balloon placement may affect prostate motion. For example, the endorectal balloon should be placed behind the prostate, pushing the prostate toward the pubic symphysis for better immobilization. If the endorectal balloon is inserted more deeply, relatively superior to the proper position, the prostate would be moved inferiorly. The improper positioning of the endorectal balloon might diminish its effect on prostate immobilization, and result in larger interfraction variability in prostate position throughout the treatment course. The presumption is compatible with the finding of van Lin et al. [20]. They evaluated the prostate motion with or without endorectal balloon placement. The day-to-day deviations (random error) in prostate position relative to adjacent bony anatomy were $2.8 \mathrm{~mm}$ and $2.5 \mathrm{~mm}$ in patients with or without endorectal balloon placement, respectively. The use of endorectal balloon did not appear to affect prostate immobilization or reduce position variation. Nevertheless, endorectal balloon placement has been shown to play a role in reducing intrafractional displacement of the prostate. The use of endorectal balloons reduced maximum intrafractional displacement in any direction from $4 \mathrm{~mm}$ to $1 \mathrm{~mm}$ [3] and the mean displacement of the prostate from $1.8 \mathrm{~mm}$ to $1.3 \mathrm{~mm}(p=0.03)$.

According to the ICRU 50 report, the planning target volume (PTV) accounts for both movement of the clinical target volume (CTV) and variations in set-up position. Stroom et al. demonstrated that the needed margin should be twice the systematic error plus 0.7 times the random error [14]. This estimated margin ensures that $99 \%$ of the CTV would receive $\geq 95 \%$ of prescribed dose. van Herk et al. also 
showed the margin should be 2.5 times the systematic error plus 0.7 times the random error, to ensure a minimum dose to the CTV of $\geq 95 \%$ of the prescribed dose for $90 \%$ of their patients [17]. In the study of internal prostate motion with endorectal balloon by van Lin et al., the systematic and random errors were $1.0 \mathrm{~mm}$ and $1.5 \mathrm{~mm}$ in the LR direction, $3.0 \mathrm{~mm}$ and $1.8 \mathrm{~mm}$ in the SI direction, and $2.5 \mathrm{~mm}$ and $2.8 \mathrm{~mm}$ in the AP direction, respectively [20]. Based on the mathematical model mentioned above, the reasonable margins to PTV are $7-9 \mathrm{~mm}$ for the SI and AP directions, and $3-4 \mathrm{~mm}$ for the LR direction with the use of endorectal balloons in prostate radiotherapy.

In this study, patients were treated in prone position with vacuum immobilization. The treatment position is a significant factor affecting set-up error and prostate motion. Stroom et al. evaluated prostate motion in patients treated in the prone and supine positions. They demonstrated that the prone position was associated with smaller random (day-to-day) variations but larger systematic variations [15]. In another study with different treatment positions for prostate radiotherapy, prostate motion in the AP direction was significantly higher in the prone position [1]. More pre-treatment corrections were required for the prone position. With careful correction before treatment, no significant difference between the supine and prone positions was seen in the isocenter positioning error. In our study, the random (interfraction) error in treatment isocenter position relative to bone was higher in the AP direction $(3.2 \mathrm{~mm}, 1 \mathrm{SD})$ compared with the SI $(2.3 \mathrm{~mm})$ and LR $(2.6 \mathrm{~mm})$ directions. These data were comparable with those of other studies $[8,11,18]$.

The mean displacements and systematic errors of isocenter relative to bony landmark in this study were relatively larger as compared to the other studies $[7,9,20]$. Similarly in the study by van Lin et al., a relatively larger systematic error was observed with the use of balloon as compared to without the use of that [20]. In the randomized trial of supine versus prone positioning, the prone position had a larger total positioning error compared with supine position, though the difference did not reach the statistical significance [1]. More pre-treatment corrections were also required in patients treated with prone position. In our study, patients were all treated with prone position and with the use of endorectal balloon. Both factors might contribute to the relatively larger systematic error in this study.

The systematic error plays a more important role in estimating the PTV margin than random error according to the mathematical model [14,17]. A larger margin for PTV is needed if a greater systematic error is present. It may subsequently result in the higher risk of radiation-induced toxicity, especially in the risk of proctitis. Based on our results, the theoretical margin for the study cohort was $8-11 \mathrm{~mm}$ in AP direction. However, the risk of rectal toxicity might be unacceptably high in this situation. Therefore, our data emphasize that more frequent image-guided set-up corrections are needed to reduce the errors.

To reduce the margins for treatment targets and the risk of radiation-related toxicity, the correction of set-up error is an important issue, especially for systematic error. In one study with the off-line correction protocol for prostate radiotherapy, the systematic displacements were reduced from 2-4 mm (1 SD) in three orthogonal directions to 1$1.5 \mathrm{~mm}$ [4]. Another study demonstrated similar findings [20]. With the off-line correction strategy based on the implanted marker, the systematic errors were effectively reduced from $2.5-4.5 \mathrm{~mm}$ to $<2 \mathrm{~mm}$. A smaller margin for PTV would be given if systematic error is reduced. It might be helpful to subsequently reduce the risk of radiation-induced complication. With the use of endorectal balloon and in prone position, the relatively larger systematic error may offset the potential benefit from the balloon and prone position. We are currently conducting another prospective study to evaluate the effectiveness of an off-line correction strategy using an on-board cone-beam CT device and more frequent image registration. The exact effect of this correction protocol is still under investigation.

To our knowledge, the issue of deformation of the endorectal balloon has seldom been addressed. As seen in Fig. 2, the shape of the endorectal balloon varied over the treatment course on EPIs. The variations in balloon shape were relatively larger in the SI direction as compared to the AP and LR directions. In half of our patients, the maximal variation in balloon diameter was in the SI direction. The presence of stool and gas beside the balloon, and the status of bladder filling are the likely causes of this larger variation. In this study, no significant correlations were found between the deformation and displacement of endorectal balloon in the SI, AP, or LR direction. It implied that the displacement of balloon was not directly due to the deformation of this device.

Deformation of the endorectal balloon might influence the status of rectal filling, subsequently affecting prostate motion. Ghilezan et al. demonstrated that status of rectal filling was the most significant predictor of intrafraction prostate motion [6]. Crook et al. also showed that the prostate position is related to rectal and bladder filling [2]. Another study also demonstrated a strong correlation between rectal volume and anterior-posterior displacement of the prostate [18]. Distension of the rectum and bladder would displace the prostate in the AP direction. Less filling allows the prostate to move more posteriorly and inferiorly. In a study of various endorectal balloons for prostate radiotherapy, variation in endorectal balloon type was associated with variation in rectal sparing [19]. The type of endorectal balloon (varying in size and shape) may be another important factor in rectal filling and prostate immobilization. Therefore, interpretation of the data on displacement should be limited to one type of endorectal balloon. Since the maximal displacement in the SI direction does not relate to balloon deformation, the set-up error from variations in daily placement depth plays the main role in this deviation.

There were some limitations in our study. The variation in the isocenter position relative to the bony landmark simply reflected the positioning set-up error, but not the exact displacement of prostate. On EPIs, the actual position of the prostate could not be determined without marker assistance. The implanted marker or in-room image device (e.g. cone-beam CT) would be helpful to more accurately measure the displacement between the endorectal balloon and the prostate. Given the institutional policy of using fiducial markers was not available during our study period, it 
remained difficult to determine the exact position of prostate in this way. In this situation, we used the contiguous bony landmark as the reference point to measure the interfraction displacement of endorectal balloon. After careful pre-treatment set-up, the bony structures may be stably positioned in daily radiation treatment, and may serve as the acceptable references for our measurement of set-up errors of endorectal balloon.

The impact of endorectal balloon on prostate immobilization has been well investigated in many studies $[3,20,21]$. These studies demonstrated the prostate motion with or without endorectal balloon, but somewhat underestimated the fraction-to-fraction variation of endorectal balloon in its daily placement. Therefore, the immobilization effect of endorectal balloon is not the key issue in our study. Our study is aimed to quantitatively determine the interfraction displacement of endorectal balloon during the whole treatment course. Based on our data, a constant protocol may be needed for daily placement of balloon as this may account for some of the differences in daily positions. To reduce the variation of daily balloon placement may further help immobilize the prostate more accurately. Another limitation in this study was the difficulty in evaluating the three-dimensional deformation of the endorectal balloon from EPIs. The EPIs showed the shape of the endorectal balloon only in lateral or AP views. To quantify the deformation, we measured variation in balloon diameter in three orthogonal directions. However, the three-dimensional deformation and volumetric change data were still unavailable. The linac-integrated cone-beam CT, imageguided radiation therapy system, may provide a solution to this problem. With the contouring on cone-beam CT image, we can evaluate the three-dimensional position displacement and volume variation in the endorectal balloon for each treatment. Moreover, the interfractional and intrafractional motion of the prostate can be directly observed using these integrated imaging devices. Further investigation of the effect of endorectal balloon placement on prostate motion should lead to the development of practical guidelines for using the endorectal balloon in prostate IMRT.

In conclusion, the random (interfraction) variation in endorectal balloon position was larger in the SI direction than in the AP and LR directions. With the use of endorectal balloon, the set-up error in treatment isocenter position relative to bone was acceptable in prostate IMRT. The deformation of the endorectal balloon was evident on EPIs taken after daily placement. Further investigations are needed to evaluate the deformation of endorectal balloons and set-up error associated with endorectal balloon placement using an image-guided radiotherapy device. Comprehensive set-up error data would be helpful in defining the satisfactory margin to achieve both adequate tumor coverage and rectum sparing.

* Corresponding author. Jason Chia-Hsien Cheng, Division of Radiation Oncology, Department of Oncology, National Taiwan University Hospital, No. 7, Chung-Shan South Road, Taipei 100, Taiwan. E-mail address: jasoncheng@ha.mc.ntu.edu.tw

Received 28 November 2006; received in revised form 28 March 2007; accepted 13 June 2007; Available online 13 August 2007

\section{References}

[1] Bayley AJ, Catton CN, Haycocks T, et al. A randomized trial of supine vs. prone positioning in patients undergoing escalated dose conformal radiotherapy for prostate cancer. Radiother Oncol 2004;70:37-44.

[2] Crook JM, Raymond Y, Salhani D, Yang H, Esche B. Prostate motion during standard radiotherapy as assessed by fiducial markers. Radiother Oncol 1995;37:35-42.

[3] D’Amico AV, Manola J, Loffredo M, et al. A practical method to achieve prostate gland immobilization and target verification for daily treatment. Int J Radiat Oncol Biol Phys 2001;51:1431-6.

[4] de Boer HC, van Os MJ, Jansen PP, Heijmen BJ. Application of the No Action Level (NAL) protocol to correct for prostate motion based on electronic portal imaging of implanted markers. Int J Radiat Oncol Biol Phys 2005;61:969-83.

[5] Dearnaley DP, Hall E, Lawrence D, et al. Phase III pilot study of dose escalation using conformal radiotherapy in prostate cancer: PSA control and side effects. $\mathrm{Br} J$ Cancer 2005;92:488-98.

[6] Ghilezan MJ, Jaffray DA, Siewerdsen JH, et al. Prostate gland motion assessed with cine-magnetic resonance imaging (cineMRI). Int J Radiat Oncol Biol Phys 2005;62:406-17.

[7] Langen KM, Jones DT. Organ motion and its management. Int J Radiat Oncol Biol Phys 2001;50:265-78.

[8] Melian E, Mageras GS, Fuks Z, et al. Variation in prostate position quantitation and implications for three-dimensional conformal treatment planning. Int J Radiat Oncol Biol Phys 1997;38:73-81.

[9] Patel RR, Orton N, Tome WA, Chappell R, Ritter MA. Rectal dose sparing with a balloon catheter and ultrasound localization in conformal radiation therapy for prostate cancer. Radiother Oncol 2003;67:285-94.

[10] Pollack A, Zagars GK, Starkschall G, et al. Prostate cancer radiation dose response: results of the M.D. Anderson phase III randomized trial. Int $\mathrm{J}$ Radiat Oncol Biol Phys 2002;53:1097-105.

[11] Roeske JC, Forman JD, Mesina CF, et al. Evaluation of changes in the size and location of the prostate, seminal vesicles, bladder, and rectum during a course of external beam radiation therapy. Int $\mathrm{J}$ Radiat Oncol Biol Phys 1995;33:1321-9.

[12] Schultheiss TE, Lee WR, Hunt MA, Hanlon AL, Peter RS, Hanks $\mathrm{GE}$. Late $\mathrm{GI}$ and $\mathrm{GU}$ complications in the treatment of prostate cancer. Int J Radiat Oncol Biol Phys 1997;37:3-11.

[13] Storey MR, Pollack A, Zagars G, Smith L, Antolak J, Rosen I. Complications from radiotherapy dose escalation in prostate cancer: preliminary results of a randomized trial. Int J Radiat Oncol Biol Phys 2000;48:635-42.

[14] Stroom JC, de Boer HC, Huizenga H, Visser AG. Inclusion of geometrical uncertainties in radiotherapy treatment planning by means of coverage probability. Int J Radiat Oncol Biol Phys 1999;43:905-19.

[15] Stroom JC, Koper PC, Korevaar GA, et al. Internal organ motion in prostate cancer patients treated in prone and supine treatment position. Radiother Oncol 1999;51:237-48.

[16] van Herk $M$. Errors and margins in radiotherapy. Semin Radiat Oncol 2004;14:52-64.

[17] van Herk M, Remeijer P, Rasch C, Lebesque JV. The probability of correct target dosage: dose-population histograms for deriving treatment margins in radiotherapy. Int J Radiat Oncol Biol Phys 2000;47:1121-35.

[18] van Herk M, Bruce A, Kroes AP, Shouman T, Touw A, Lebesque JV. Quantification of organ motion during conformal radiotherapy of the prostate by three dimensional image registration. Int J Radiat Oncol Biol Phys 1995;33:1311-20. 
[19] van Lin EN, Hoffmann AL, van Kollenburg P, Leer JW, Visser AG. Rectal wall sparing effect of three different endorectal balloons in 3D conformal and IMRT prostate radiotherapy. Int J Radiat Oncol Biol Phys 2005;63:565-76.

[20] van Lin EN, van der Vight LP, Witjes JA, Huisman HJ, Leer JW, Visser AG. The effect of an endorectal balloon and off-line correction on the interfraction systematic and random prostate position variations: a comparative study. Int J Radiat Oncol Biol Phys 2005;61:278-88.

[21] Wachter S, Gerstner N, Dorner D, et al. The influence of a rectal balloon tube as internal immobilization device on variations of volumes and dose-volume histograms during treatment course of conformal radiotherapy for prostate cancer. Int J Radiat Oncol Biol Phys 2002;52:91-100.

[22] Zelefsky MJ, Fuks $Z$, Hunt $M$, et al. High dose radiation delivered by intensity modulated conformal radiotherapy improves the outcome of localized prostate cancer. J Urol 2001;166:876-81.

[23] Zietman AL, DeSilvio ML, Slater JD, et al. Comparison of conventional-dose vs high-dose conformal radiation therapy in clinically localized adenocarcinoma of the prostate: a randomized controlled trial. JAMA 2005;294:1233-9. 